\title{
Image Guided Radiotherapy of the Prostate
}

\author{
David Jaffray ${ }^{1}$, Marcel van Herk², Joos Lebesque ${ }^{2}$, and Alvaro Martinez ${ }^{1}$ \\ ${ }^{1}$ William Beaumont Hospital, Royal Oak, MI USA \\ djaffray@beaumont .edu \\ ${ }^{2}$ Radiotherapy Department, The Netherlands Cancer Institute, Amsterdam, The Netherlands \\ portal@nki.nl
}

\begin{abstract}
This paper describes the prototype of an integrated system for conebeam CT guided radiotherapy of prostate cancer. The system works by acquiring a 3D image set just prior to treatment delivery. This image set is matched automatically with bones and prostate defined in the planning CT to determine the required couch translation and to determine a best fitting treatment plan which is subsequently delivered. Because of the improved localization of the prostate on the treatment machine, potentially smaller safety margins can be applied and a higher dose can be delivered leading to a higher cure rate without increasing damage to healthy tissues.
\end{abstract}

\section{Introduction}

External beam radiotherapy aims at killing a tumor with high-energy photons, while sparing as much as possible surrounding healthy tissues. In general, the treatment is planned on CT and/or MR images, and it is delivered in daily fractions over a period of several weeks. To focus the radiation on the required spot, individually shaped beams are delivered from multiple directions by a small linear accelerator mounted in a rotating gantry. A complicating factor is the limited precision of tumor localization on the treatment machine, due to tumor delineation inaccuracies, movement of the tumor within the body and variations in daily positioning of the patient. For this reason, a safety margin must be applied, i.e., a larger volume is treated than the actual tumor to compensate for geometric inaccuracies [e.g., 11]. As the precision increases, the safety margin can be reduced and a higher dose can be given to the target without unacceptable damage to surrounding healthy tissues.

Several methods have been investigated to improve the precision of prostate irradiation. Electronic portal imaging, imaging the patient with the treatment beam, has been shown successful in measuring and reducing the errors in repositioning of the bony anatomy [e.g., 1,3]. More recently, ultrasound imaging has been applied to improve the localization of the prostate on the treatment machine [e.g., 8]. Another localization approach that has been explored is X-ray radiography of metal markers implanted into the prostate [7] (projected radiography has insufficient contrast to visualize the prostate directly). The aim of this project is to develop an integrated delivery system that performs three-dimensional localization of the prostate on the treatment machine just prior to treatment delivery. The system has the following re-

W. Niessen and M. Viergever (Eds.): MICCAI 2001, LNCS 2208, pp. 1075-1080, 2001.

(C) Springer-Verlag Berlin Heidelberg 2001 
quirements. The technique must not be invasive (no markers) and must not require physical contact with the patient to avoid deformation of soft tissues in the pelvis (this happens with ultrasound localization). Furthermore the localization, analysis and treatment process should be as fast as possible to maintain an acceptable patient throughput and in particular to reduce the probability of patient or tumor movement in the time interval between imaging and treatment.

\section{Material and Methods}

A cone beam CT scan will be acquired on the treatment machine for each treatment fraction (Fig. 1). This 3D CT data set is analyzed automatically to localize the prostate and the treatment prescription is adapted to match the actual position and orientation of the prostate. Finally, the treatment is delivered.
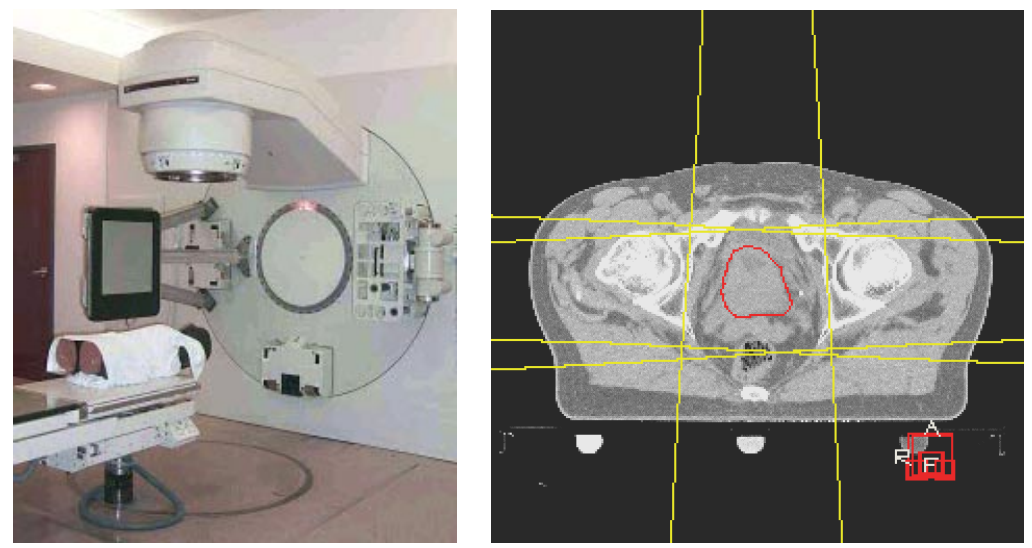

Figure 1. (a) Prototype cone beam CT scanner mounted on the gantry of a commercial medical linear accelerator. A diagnostic X-ray tube (right) and a flat panel imager (left) have been added. (b) Conventional treatment planning is based on delineation of the target in 3D. Multiple beams are planned that encompass the target with a safety margin for geometrical errors.

Image acquisition. The cornerstone of the system is a kilovoltage cone-beam CT system that is integrated on the gantry of an Elekta SL-20 linear accelerator [4]. The radiation source of the system is a diagnostic $\mathrm{x}$-ray tube, with its beam axis perpendicular to the treatment beam. Opposite the tube is a $41 \mathrm{x} 41 \mathrm{~cm}$ flat panel imaging device (consisting of a fluorescent screen coupled to an 1024×1024 pixel amorphous silicon photodiode array on a glass substrate), mounted at $160 \mathrm{~cm}$ from the isocenter. To obtain sufficient field of view to image the whole pelvis, the detector will be offset by $20 \mathrm{~cm}$, and half field images are made over 360 degrees of rotation in a single gantry rotation.

A three-dimensional image of the patient is obtained by cone beam reconstruction using the Feldkamp algorithm for limited cone reconstruction [2]. Because of the retractable construction of the imaging system, there is a substantial flex of the com- 
ponents as function of gantry rotation. This flex is reproducible and has been taken into account in the reconstruction algorithm to obtain a high image quality [5].

Image analysis. CT-based treatment planning in a routine clinical setting typically takes several hours per patient (Fig. 1b). On-line application, i.e., while the patient is on the treatment table, requires considerable acceleration of this process. The model that will be applied is based on the observation that the movement of the prostate relative to the pelvis can be well described by a rotation and translation, where most rotation occurs around the left-right axis of the patient [10]. The proposed image analysis algorithm first registers the segmented bony anatomy of the on-line CT scan with a planning CT scan using a chamfer matching algorithm [9]. Then the displacement of the prostate relative to the pelvis is measured using a registration algorithm similar to the one developed by Woods et al [13]. For this purpose, prostate contours are manually defined in the planning CT, which is also part of planning for conventional radiotherapy. The planned prostate shape is then aligned to the on-line CT by minimization of the variance of the gray values of the on-line CT under the predefined prostate shape. The minimum corresponds with the rotation and translation of the prostate from its position in the initial treatment plan.

Treatment planning, delivery and verification. Existing treatment planning technology can be employed in a novel manner to minimize the on-line planning time by identifying the most probable degrees of prostate motion and preparing a set of plans to accommodate this motion. First, the patient couch is translated to account for translation of bone and prostate relative to the treatment room. Then a best fitting treatment plan is selected from the set of pre-defined treatment plans to account for prostate rotation.

The selected treatment plan will be delivered by means of a step-and-shoot intensity delivery technique using 5 beam angles and about 20 treatment segments. The beam shaping occurs by means of a standard multi-leaf collimator.

During treatment, regular verification such as electronic portal imaging and in-vivo dosimetry will be performed. In addition, cone beam acquisition is repeated after treatment delivery. Off-line re-planning based on the pre- and post-treatment CT images will be performed to determine the cumulative radiation dose delivered to prostate and critical structures. For this purpose, the prostate shape and position will be interpolated between the pre- and post-treatment scans. Because the off-line planning is performed under less time pressure, the progress of the treatment will be more carefully monitored compared to the on-line procedure. In addition, the safety is increased because possible deficiencies in each fraction can be rectified on later fractions.

\section{Results}

Image acquisition. Prototypes of the hardware and software of the system have been constructed. Cone-beam CT of anthropomorphic phantoms has been acquired on the treatment machine, with an acquisition time of 3 minutes (Fig. 2), but a reconstruction time of hours. The final system will acquire a scan in 1 minute and will use a hardware 
accelerated reconstruction system. With flex calibration, the image quality of the cone beam CT data is similar to a diagnostic CT scan.
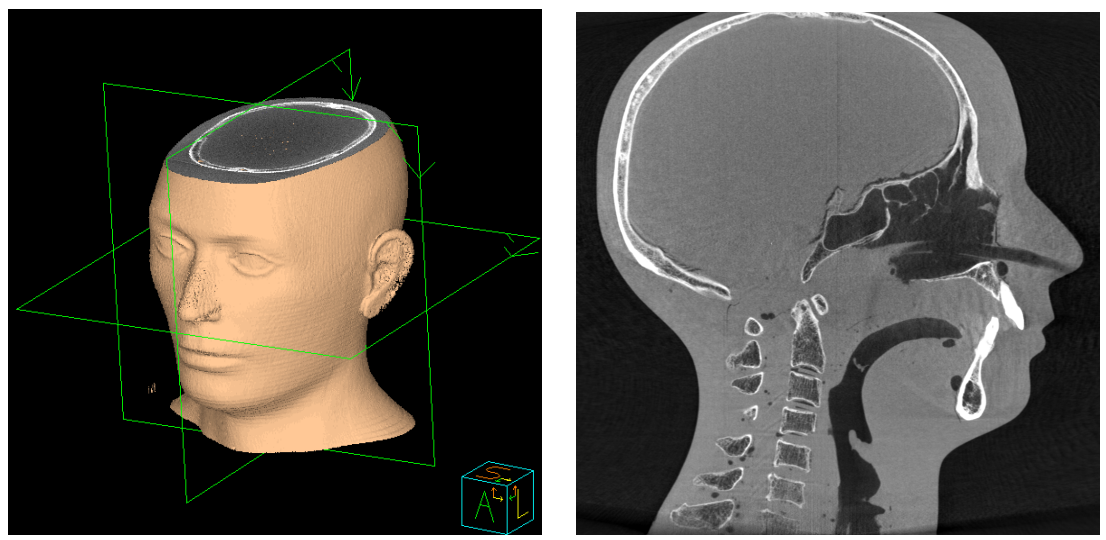

Figure 2. Cone-beam reconstruction of a head phantom acquired with the cone beam CT scanner of Fig. 1. The image was made with 320 projections at $120 \mathrm{kVp}$ and $200 \mathrm{mAs}$ in $180 \mathrm{~s}$. (a) Surface rendering with axial slice. (b) Sagittal cut. The image quality is similar to a diagnostic CT scan.

Image analysis. The results of the proposed algorithm for on-line prostate localization are shown in Figs. 3a-d. In the baseline CT, the pelvic bone and the prostate are predefined. On-line matching of the planning CT with the proposed cone-beam CT (both with calibrated isocenter positions) on the pelvic bone determines the setup error (translation and rotation) of the bony anatomy. Figs. 3a and $b$ represent on-line CTs and have been taken from an organ motion study, matched in this way to the planning CT. The motion of the prostate is apparent because the prostate contours (dotted white line), which were delineated in the planning CT, do not fit the prostate in these 'online' images. The motion of the prostate relative to the bony anatomy is quantified by translating and rotating the prostate contours until they cover a homogeneous area, i.e., an area with minimal standard deviation of gray values (Figs $3 \mathrm{~b}$ and c). The flow chart in Fig. 3e illustrates the algorithm. The gray boxes correspond to a-priori available information. Because the bone matching can be performed accurately on fairly low resolution $\mathrm{CT}$ and the prostate matching only requires a small part of the conebeam $\mathrm{CT}$ volume, the complete image analysis can be performed in less than $10 \mathrm{~s}$ on a $700 \mathrm{MHz}$ PC.

For this particular problem, this procedure corresponds closely with that of manual delineation. It is important to note that in the case of CT, such algorithms do not necessarily localize the prostate, but rather the contrasting structure defined in the 3D template. Although this is the best result that can be obtained using CT data, any systematic deviations between the actual prostate location (defined best by MR) and the CT-defined prostate must be considered separately $[6,11]$. 

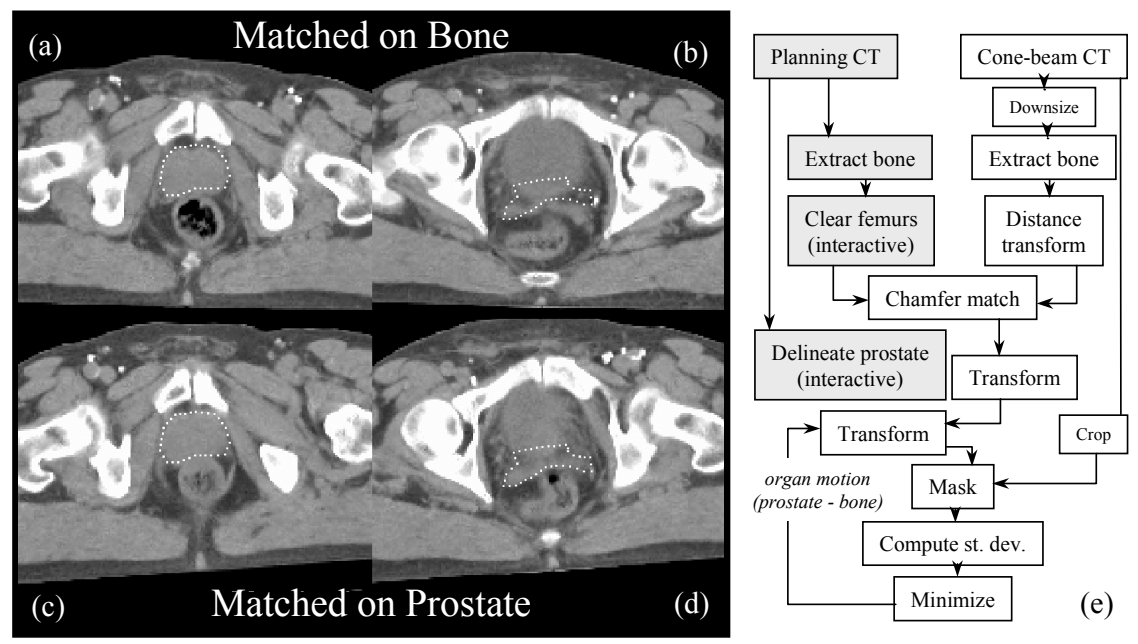

Figure 3. Automated localization of the prostate. $(a, b)$ Reference prostate contour overlaid on the "on-line" CT based on registration to bony anatomy. This transformation is adjusted using Woods' algorithm to locate the prostate in the on-line set (c,d). Much improved coverage of the prostate is thus achieved. (e) Flow-chart summary of the algorithm (priors are gray).

\section{Discussion and Conclusions}

We implemented a system for cone beam CT on a clinical accelerator. The phantom tests show excellent spatial resolution and contrast to noise performance. The image quality is adequate to resolve tumors visible on diagnostic CT. A software system has been developed for image reconstruction and processing. The image analysis is very fast, but the cone beam CT reconstruction is at present prohibitively slow (hours). In the final system, a specialized hardware accelerator will be applied to reduce the reconstruction time to 1 minute. One problem that needs to be addressed in more detail is the mobility of the patient and his organs on the treatment machine in the time between image acquisition and delivery. We will perform repeat MRI studies on volunteers and patients to determine the time dependent statistics of prostate movement. The results will determine the amount of margin reduction that can safely be achieved with this approach.

\section{References}

1. Bel, A., van Herk, M., Bartelink, H., Lebesque, J.V.: A verification procedure to improve patient set-up accuracy using portal images. Radiother. Oncol. 29 (1993) 253-260

2. Feldkamp, L.A., Davis, L.C. and Kress, J.W.: Practical cone-beam algorithm. J. Opt. Soc. Am. A. 1 (1984) 612-619 
3. Gilhuijs, K.G.A., van der Ven, P.J.H. and van Herk, M.: Automatic three-dimensional inspection of patient setup in radiation therapy using portal images, simulator images, and computed tomography data. Med. Phys. 23 (1996) 389-399

4. Jaffray, D.A., Drake, D.G., Moreau, M., Martinez, A.A., and Wong, J.W.: A radiographic and tomographic imaging system integrated into a medical linear accelerator for localization of bone and soft-tissue targets. Int. J. Radiat. Oncol. Biol. Phys. 45 (1999) 773-789

5. Jaffray, D.A. and Siewerdsen, J.H.: Cone-beam computed tomography with a flat-panel imager: initial performance characterization. Med. Phys. 27 (2000) 1311-1323

6. Rasch, C., Barillot, I., Remeijer, P., Touw., A., van Herk, M. and Lebesque, J.V.: Definition of the prostate in CT and MRI: a multi-observer study. Int. J. Radiat. Oncol. Biol. Phys.43 (1999) 57-66

7. Shirato, H., Shimizu, S., Kunieda, T., Kitamura, K., van Herk, M., Kagei, K., Nishioka, T., Hashimoto, S., Fujita, K., Aoyama, H., Tsuchiya, K., Kudo, K., Miyasaka, K.: Physical aspects of a real-time tumor-tracking system for gated radiotherapy. Int. J. Radiat. Oncol. Biol. Phys. 48 (2000) 1187-1195

8. Troccaz, J., Laieb, N., Vassal, P., Menguy, Y., Cinquin, P., Bolla, M., Giraud, J.Y.: Patient setup optimization for external conformal radiotherapy. J. Image. Guid. Surg. 2 (1995) 113 120

9. van Herk, M. and Kooy, H.M.: Automatic three-dimensional correlation of CT-CT, CT-MRI, and CT-SPECT using chamfer matching. Med. Phys. 21 (1994) 1163-1178

10. van Herk, M., Bruce, A., Kroes, A.P., Shouman, T., Touw, A., Lebesque, J.V.: Quantification of organ motion during conformal radiotherapy of the prostate by three dimensional image registration. Int. J. Radiat. Oncol. Biol. Phys. 33 (1995) 1311-1320

11. van Herk, M., de Munck, J.C., Lebesque, J.V., Muller, S., Rasch, C. and Touw, A.: Automatic registration of pelvic computed tomography data and magnetic resonance scans including a full circle method for quantitative accuracy evaluation. Med. Phys. 25 (1998) 2054-2067

12. van Herk, M. Remeijer, P. Rasch, C. Lebesque, J.V.: The probability of correct target dosage: dose-population histograms for deriving treatment margins in radiotherapy. Int. J. Radiat. Oncol. Biol. Phys. 47 (2000) 1121-1135

13. Woods, R.P., Mazziotta, J.C. and Cherry, S.R.: MRI-PET registration with automated algorithm. J. Comput. Assist. Tomogr. 17 (1993) 536-546 